ISSUES IN MEDICINE

\title{
Life-threatening Listeria meningitis: Need for revision of South African acute bacterial meningitis treatment guidelines
}

\author{
C-M Schutte, ${ }^{1} \mathrm{MB}$ ChB, MMed (Neurology), MD; C H van der Meyden, ${ }^{1} \mathrm{MB}$ ChB, FCN, MD; M Kakaza, ${ }^{1} \mathrm{MB}$ ChB, MMed (Neurology); \\ Z Lockhat, ${ }^{2} \mathrm{MB}$ ChB, FC Rad (D) SA; E van der Walt, ${ }^{2} \mathrm{MB}$ ChB, FC Rad (D) SA
}

${ }^{1}$ Department of Neurology, School of Medicine, Faculty of Health Sciences, University of Pretoria, South Africa

${ }^{2}$ Department of Radiology, School of Medicine, Faculty of Health Sciences, University of Pretoria, South Africa

Corresponding author: C-M Schutte (cschutte@medic.up.ac.za)

Background. The recent listeriosis outbreak in South Africa (SA) received widespread attention in the media. More than 1000 laboratoryconfirmed cases of listeriosis occurred during an 18 -month period, with a case fatality rate of $28 \%$. Acute bacterial meningitis due to listeriosis was extremely rare at Steve Biko Academic Hospital in Pretoria until 2017/18, when we saw two very sick adults with this condition during the listeriosis outbreak.

Objectives. To describe the presentation, treatment and outcome of these patients to raise awareness of this potentially fatal but treatable infection that does not respond to empirical third-generation cephalosporins.

Case reports. Case 1: A 60-year-old man collapsed at home after being discharged from hospital for treatment of Listeria meningitis. On readmission he had neck stiffness and a depressed level of consciousness with right-sided hemiparesis. A computed tomography (CT) scan of the brain showed possible subarachnoid haemorrhage, but on CT angio- and venograms, extensive thrombosis of the superior sagittal, right transverse and bilateral sigmoid sinuses extending into the right internal jugular vein was noted. Patient 2: A 55-year-old HIV-positive hypertensive man on highly active antiretroviral therapy and antihypertensives visited the emergency department complaining of a newonset headache. He was discharged on pain medication, but was readmitted the next day with a depressed level of consciousness, neck stiffness, low-grade fever and generalised tonic-clonic convulsions. A lumbar puncture revealed active cerebrospinal fluid that was culturepositive for L. monocytogenes. The patients received ampicillin and gentamicin for 3 weeks; the cerebral venous thrombosis was treated with unfractionated heparin. In both cases, the course of the disease was complicated. The first patient remained confused and suffered from psychotic episodes for 5 weeks. He was finally discharged after 6 weeks in hospital and continued to improve to the extent that he was able to return to work. The second patient needed intubation and ventilation and was treated in the intensive care unit. He improved over the next week and was finally discharged home with no residual neurological sequelae.

Conclusions. Our two cases demonstrate that the listeriosis outbreak should change the way we view bacterial meningitis in SA: according to the National Institute for Communicable Diseases, empirical treatment for meningitis should include ampicillin and gentamicin in all adult patients with features of meningitis. There may be a need for an updated meningitis treatment guideline in SA.

S Afr Med J 2019;109(5):296-298. DOI:10.7196/SAMJ.2019.v109i5.13866

The recent listeriosis outbreak in South Africa (SA), in particular in Gauteng Province, received widespread attention in the media. During an 18-month period, $>1000$ laboratory-confirmed cases of listeriosis occurred, with a case fatality rate of $28 \% .{ }^{[1]}$ Most infections were in neonates, the elderly and immunocompromised persons. ${ }^{[2]}$ At Steve Biko Academic Hospital in Pretoria, meningitis caused by Listeria was extremely uncommon up to 2018, but during the outbreak this condition was diagnosed in two very sick adult patients within a 9-month period. The objective of this report is to describe the presentation, treatment and outcome of these cases to raise awareness of this potentially fatal but treatable infection, which does not respond to empirical third-generation cephalosporins.

\section{Case reports}

\section{Case 1}

A 60-year-old man with no previous comorbidities presented with headache to a peripheral hospital, where a lumbar puncture confirmed active cerebrospinal fluid. He was referred to a tertiary hospital and a diagnosis of Listeria infection was made. He was treated with ampicillin and gentamicin for 10 days and improved considerably. However, the day after discharge he collapsed and fell at home and was taken to Steve Biko Academic Hospital. On arrival, he complained of severe headache and had a laceration on the back of his head, neck stiffness and a mild right-sided hemiparesis with increased tone in all four limbs.

An urgent computed tomography (CT) scan of the brain revealed evidence of a possible subarachnoid haemorrhage, and a CT angiogram was requested (Fig. 1). This revealed thrombosis of the superior sagittal, right transverse and bilateral sigmoid sinuses that extended into the right internal jugular vein. A left occipital lobe hypodensity was noted, indicating a subacute stroke; analysis of the cerebrospinal fluid (CSF) showed a high protein level of $0.56 \mathrm{~g} / \mathrm{L}$, 4 neutrophils, normal glucose and xanthochromia. Culture of the CSF did not yield any bacterial growth.

The patient was treated with intravenous ampicillin, gentamicin and ceftriaxone for 3 weeks and low-molecular-weight heparin was 

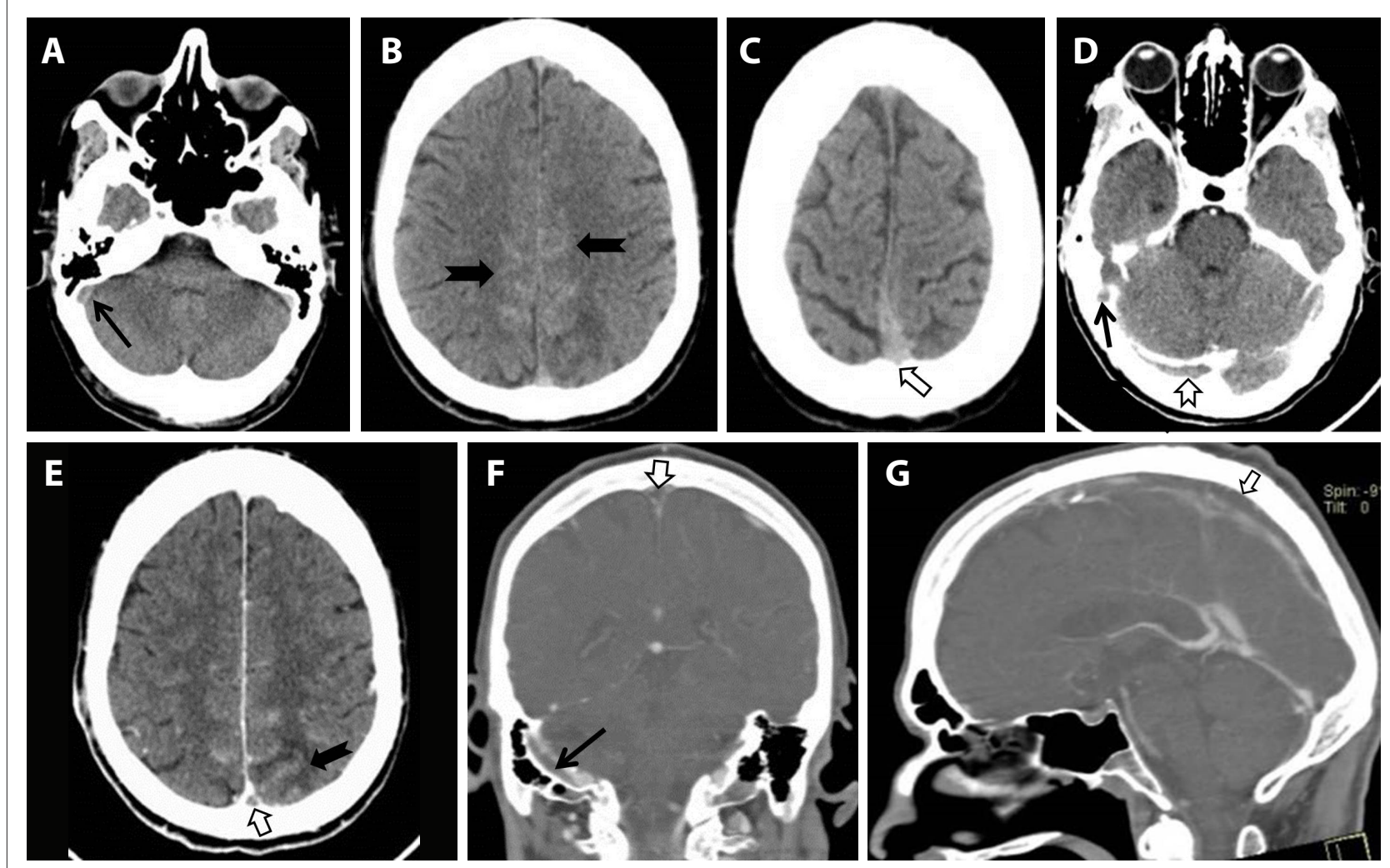

Fig. 1. Pre-contrast axial images demonstrate high density in the right sigmoid sinus (A, black arrow), cortical veins (B, black arrows) and superior sagittal sinus (C, white arrow). Post-contrast axial images demonstrate filling defects in the right sigmoid ( $D$, black arrow), transverse ( $D$, white arrow) and superior sagittal sinus (E, white arrow), indicating thrombosis. Oedema is noted in the centrum semi-ovale, and enhancement in the cortical veins could be mistaken for haemorrhage (E, black arrow). Post-contrast coronal $(F)$ and sagittal $(G)$ images demonstrate filling defects in the right sigmoid (black arrow) and superior sagittal sinus (white arrows).

administered (first daily, then as a twice-daily dose of $40 \mathrm{mg}$, then again daily). He had a difficult course in the ward, complicated by confusion and psychosis that was challenging to manage. A repeat CT angio- and venogram still showed venous sinus thrombosis as before, with improvement in the size of the occipital lobe infarct. Electroencephalography did not show evidence of subclinical status epilepticus or any other seizure activity; the dominant background rhythm showed slow frequencies in the theta and delta ranges, indicating a diffuse cerebral dysfunction. The patient was co-managed by the neurology and psychiatry departments and was finally discharged home after 6 weeks. He continued to improve and was well on follow-up, having returned to his work after some weeks.

\section{Case 2}

A 55-year-old HIV-positive hypertensive man on highly active antiretroviral therapy and antihypertensive medication complained of a new-onset headache for which he visited the emergency department. His vital parameters were all normal and he was sent home on pain medication. Twenty-four hours later he presented again, now with a depressed level of consciousness, neck stiffness and a low-grade fever. The Glasgow Coma Scale was recorded as $11 / 15$ and the patient had generalised tonic-clonic convulsions in the emergency unit that were aborted with intravenous benzodiazepines.

A CT scan of the brain showed loss of the grey-white matter interface and flattened sulci, indicating mild brain oedema but no evidence of any focal lesions. Lumbar puncture showed a neutrophil count of $410 / \mu \mathrm{L}$, a lymphocyte count of $30 / \mu \mathrm{L}$, a protein level of
$3.62 \mathrm{~g} / \mathrm{L}$ and a glucose level of $2.2 \mathrm{mmol} / \mathrm{L}$; the serum glucose level was $9 \mathrm{mmol} / \mathrm{L}$. On Gram stain, scanty Gram-positive bacilli were noted. The patient's level of consciousness deteriorated further and he was intubated and ventilated in the intensive care unit.

Empirical treatment for meningitis with ceftriaxone, ampicillin and gentamicin was started. After 3 days the CSF culture came back positive for L. monocytogenes. The patient improved steadily and was able to move to the general neurology ward after 1 week. He was discharged with no obvious neurological deficits after 1 month in hospital.

\section{Discussion}

In December 2017, the Minister of Health informed the public of an outbreak of listeriosis in SA, with the majority of cases being seen in Gauteng. Infection by Listeria became a notifiable disease in SA, and by January 2018, 727 laboratory-confirmed cases of infection had been documented, with $>60 \%$ occurring in Gauteng, $13 \%$ in the Western Cape and 7\% in KwaZulu-Natal. By July 2018, 1060 cases had been reported and 216 patients had died, making this the world's worst documented outbreak of listeriosis. ${ }^{[3]}$ Reported numbers of new cases declined steadily after the recall of suspected food items from March 2018. Of all clinical isolates taken from ill patients with listeriosis, 92\% belonged to Listeria sequence type 6 (ST6) and were genetically similar, confirming that a single strain of Listeria was responsible for the outbreak. ${ }^{[3]}$

Reports from the world literature on meningitis mention Listeria infection as the third most common cause of acute bacterial 
meningitis ${ }^{[4,5]}$ yet during the past decade meningitis caused by L. monocytogenes was extremely rare in the adult neurology unit at Steve Biko Academic Hospital. Guidelines issued by the Infectious Diseases Society of Southern Africa 6 years ago stated that empirical treatment with ampicillin for possible Listeria meningitis was indicated only in patients aged $>50$ years, or those who were immunocompromised because of immunosuppressive drugs, alcoholism, liver cirrhosis, asplenia, end-stage renal failure or diabetes mellitus. ${ }^{[6]}$ HIV infection was not seen as an indication for ampicillin use. This situation has changed completely after the listeriosis outbreak of 2017/18, where empirical ampicillin and gentamicin was advocated in all adult patients who presented with features of acute meningitis. The National Institute for Communicable Diseases advised that 'all adults who present with signs and symptoms of meningitis be initiated on a third-generation cephalosporin AND ampicillin plus gentamicin, at doses appropriate for the treatment of meningitis. ${ }^{\text {[7] }}$

In the world literature, untreated central nervous system listeriosis has been reported to have a $100 \%$ mortality, and in treated cases mortality ranges from $13 \%$ to $>40 \%{ }^{[8,9]}$ With a diagnosis of encephalitis in a patient with CNS listeriosis, mortality increases significantly. ${ }^{[10]}$ Brainstem encephalitis (rhombencephalitis) secondary to Listeria infection is a serious condition that may occur in both immunocompetent and immunocompromised patients. We have not encountered this condition in our unit for the past 5 years, and our two patients did not show signs of brainstem involvement. However, an SA case series from the Western Cape published in $2015^{[11]}$ described six patients with brainstem encephalitis; at that time the authors postulated that three of their cases could have been related to a food-borne mini-outbreak, since the patients presented within 6 months of one another and came from the same geographical area. Two of these patients had adverse outcomes. ${ }^{[11]}$

Other risk factors for adverse outcomes have been reported to include non-haematological malignancy, alcoholism, age $\geq 70$ years, cortisone use and kidney disease. ${ }^{[12]}$ Although one of our patients was immunocompromised due to HIV infection, neither had any of these risk factors, and both patients fortunately eventually had a good outcome. Interestingly, research from The Netherlands showed an increase in mortality in patients with Listeria meningitis when a 2006 - 2012 study was compared with an earlier study from 1998 to 2002; this was attributed to a change in the genotype sequence type of L. monocytogenes. During the earlier period, only $4 \%$ of patients had CNS infection caused by L. monocytogenes type 6 (ST6), but in the later period, almost a third of cases were due to this sequence type, indicating that it may be particularly aggressive. ${ }^{[13]}$ As mentioned above, this was the serotype of Listeria that was implicated in the SA outbreak.

Interestingly, as far as we are aware the complication of cerebral venous sinus thrombosis secondary to Listeria meningitis has not been described previously. Our first patient initially seemed to make a good recovery from his infection, but then suddenly deteriorated and collapsed just after discharge from hospital. Cerebral venous sinus thrombosis can be a difficult condition to diagnose, as we found in our patient, in whom the initial diagnosis was a subarachnoid haemorrhage. Further complications of cerebral venous thrombosis include venous infarcts and subsequent haemorrhage, as well as thrombus extension. ${ }^{[14]}$ Our patient did have evidence of possible bleeding as reflected by the xanthochromia in the CSF, as well as evidence of a venous infarct as indicated by the occipital low-density area on CT of the brain.

In September 2018, the Minister of Health announced that the listeriosis outbreak in SA was over, since the number of new cases reported was very low. ${ }^{[15]}$ However, our second patient presented in October 2018, indicating that new cases will probably continue to emerge for some time, in part owing to the long incubation period of Listeria in some individuals. We therefore caution against complacency.

\section{Conclusions}

Our two cases demonstrate that the listeriosis outbreak should change the way we view bacterial meningitis in SA. According to the National Institute for Communicable Diseases, empirical treatment for meningitis should include ampicillin and gentamicin in all adult patients with features of meningitis, but the question remains whether clinicians should now return to the old guidelines as the outbreak is reported to be over, or the abovementioned suggestions (third-generation cephalosporin plus ampicillin and gentamicin) should be implemented into national guidelines for the empirical treatment of acute bacterial meningitis. In addition, clinicians should have a very high index of suspicion when any adult presents with a new-onset headache, even in the absence of fever and systemic signs of infection.

\section{Declaration. None.}

Acknowledgements. None.

Author contributions. C-MS wrote the article, CHvdM and MK helped with critical appraisal and literature reviews, and ZL and EvdW helped with the radiological reporting and reviewing.

Funding. None.

Conflicts of interest. None.

1. National Department of Health, South Africa. National public health emergency response plan: Listeriosis 2017/18. 23 April 2018. http://www.health.gov.za/index.php/component/ phocadownload/category/439 (accessed 28 January 2019).

2. National Institute for Communicable Diseases, South Africa. NICD Listeria outbreak, situational report 27 December 2017. http://www.nicd.ac.za/index.php/nicd-listeria-outbreak-situationalreport-27-december-2017/ (accessed 28 January 2019).

3. National Department of Health, South Africa. Listeriosis outbreak, situational report 26 July 2018. http://www.nicd.ac.za/wp-content/uploads/2018/07/Listeriosis-outbreak-situation-report _26July2018_fordistribution.pdf (accessed 28 January 2019).

4. Brouwer MC, van de Beek D, Heckenberg SGB, Spanjaard L, de Gans J. Community-acquired Listeria monocytogenes meningitis in adults. Clin Infect Dis 2006;43(10):1233-1238. https://doi. org $/ 10.1086 / 508462$

5. Sigurdardóttir B, Björnsson MO, Jónsdóttir KE, Erlendsdóttir H, Gudmundsson S. Acute bacterial meningitis in adults: A 20-year overview. Arch Intern Med 1997;157(4):425-430. https://doi. org/10.1001/archinte. 1997.00440250077009

6. Boyles $\mathrm{TH}$, Buffor $\mathrm{C}$. Ba children and adults in South Africa. South Afr J Epidemiol Infect 2013;28(1):5-15. https://doi.org
chengitis children and adults in South Afr

National Institute for Communicable Diseases, South Africa. Increase in cases of Listeria meningitis. http://www.nicd.ac.za/index.php/increase-in-cases-of-listeria-meningitis/ (accessed 28 January
2019). 2019).

8. Charlier C, Perrodeau É, Leclercq A, et al.; MONALISA study group. Clinical features and prognostic factors of listeriosis: The MONALISA national prospective cohort study. Lancet Infect Dis 2017;17(5):510-519. https://doi.org/10.1016/S1473-3099(16)30521-7

9. Skogberg K, Syrjänen J, Jahkola M, et al. Clinical presentation and outcome of listeriosis in patients with and without immunosuppressive therapy. Clin Infect Dis 1992;14(4):815-821. https://doi. org/10.1093/clinids/14.4.815

10. Armstrong RW, Fung PC. Brainstem encephalitis (rhombencephalitis) due to Listeria monocytogenes: Case report and review. Clin Infect Dis 1993;16(5):689-702. https://doi.org/10.1093/clind/16.5.689

1. Fredericks P, Britz M, Eastman R, Carr JA, Bateman KJ. Listerial brainstem encephalitis - treatable, but easily missed. S Afr Med J 2015;105(1):17-20. https://doi.org/10.7196/SAMJ.8700

12. Guevara RE, Mascola L, Sorvillo F. Risk factors for mortality among patients with nonperinatal Guevara RE, Mascola L, Sorvillo F. Risk factors for mortality among patients with nonperinatal
listeriosis in Los Angeles County, 1992 - 2004. Clin Infect Dis 2009;48(11):1507-1515. https://doi.

13. Koopmans MM, Brouwer MC, Bijlsma MW, et al. Listeria monocytogenes sequence type 6 and increased rate of unfavorable outcome in meningitis: Epidemiologic cohort study. Clin Infect Dis 2013;57(2):247-253. https://doi.org/10.1093/cid/cit250

14. Dentali F, Ageno W. Natural history of cerebral vein thrombosis. Curr Opin Pulm Med 2007;13(5):372-376. https://doi.org/10.1097/MCP.0b013e3282058b81

15. National Department of Health, South Africa. Media statement by the Minister of Health on listeriosis outbreak. 3 September 2018. http://www.nicd.ac.za/wp-content/uploads/2018/09/Mediastatement-on-listeriosis-1-3.pdf (accessed 28 January 2019). 